\title{
Effects of age, antigen deprivation, and allograft rejection on epithelial cell kinetics in mouse colon
}

\author{
R. J. HOLDEN ${ }^{1}$ AND ANNE FERGUSON \\ From the University Departments of Medicine and of Bacteriology and Immunology, Western Infirmary, \\ Glasgow
}

SUMMARY A technique of microdissection of colonic mucosa has allowed the study of mitotic activity, measured by metaphase accumulation following colchicine blockade, in individual crypts of mouse colon. The changes occurring during growth and development of normal colon have been studied and compared with changes found in antigen free colon (colonic isografts) and in cell-mediated immune damage of the bowel (allograft rejection). Metaphase accumulation was steady at two metaphases per hour in baby mouse colon until 18 days after birth. Between 18 and 24 days a rapid, and significant increase in mitotic activity occurred $(\mathrm{P}<0.01)$, reached adult values, and changed no further. Metaphase accumulation in isografts was similar to normal colon for the first two weeks after transplantation but the rise in mitotic activity in the third week did not occur. Allografts of colon showed two- to three-fold increases in metaphase accumulation when compared with both normal colon and isografts $(P<0.01)$. When crypt mitotic activity was compared with the length of crypts measured in histological sections of normal colon, isografts, and allografts, no clear relationship was observed. Both changes in the luminal environment of the gut at the time of weaning and cell-mediated immune reactions in the colonic wall appeared to be associated with increased mitotic activity in colonic crypts.

The epithelial cell kinetics of the gastrointestinal tract mucosae maintain a steady state with cell renewal balanced by cell loss. In the stomach the proliferative region is in the neck of the gastric glands and supplies cells which migrate upwards to mature as surface mucous cells and downwards to mature as chief and parietal cells. In the small intestine and colon, the proliferative region is in the lower part of the crypt and the cells migrate upwards towards the surface as they mature (Lipkin, 1973). In a previous report (Holden and Ferguson, 1976), we described the morphological development of normal mouse colon, and the effects of an antigenfree state (isografts of mouse colon) and local cellmediated immune damage (rejection of colonic allografts) on morphology. The effects of a local cell-mediated immune reaction on the colon were contrasted with those of similarly induced damage to the small intestine. In mouse small intestine, rejection produces crypt hyperplasia and shortening of the villi, so that the architecture of the mucosa resembles that of untreated coeliac disease or severe

\footnotetext{
${ }^{1}$ Address for correspondence: Dr R. J. Holden, Monklands District General Hospital, Monkscourt Avenue, Airdrie, Scotland.
}

Received for publication 11 October 1978 parasitic infection (Ferguson and Parrott, 1973; MacDonald and Ferguson, 1976; MacDonald and Ferguson, 1977; Ferguson and MacDonald, 1977). However, colonic allografts showed no evidence of crypt hyperplasia and crypts were shorter than in normal colon of the same age, suggesting possibly an atrophic or hypoplastic state induced by the cellmediated immune reaction.

Epithelial cell kinetics of small intestine can be examined by microdissection of crypts and villi after colchicine-induced metaphase arrest (Clarke, 1971). We have modified this technique to allow examination of individual crypts of colon, and this has enabled us to define the effects of age, of the normal luminal contents, and of the immunological damage of a cell-mediated immune reaction on cell kinetics in colonic mucosal crypts.

\section{Methods}

Inbred mice of the strains CBA $\left(\mathrm{H}-2^{\mathrm{k}}\right)$ and $\mathrm{Balb} / \mathrm{c}$ $\left(\mathrm{H}-2^{\mathrm{d}}\right)$, free of parasitic infections, were used. Isografts and allografts were prepared by implanting fragments of foetal colon beneath the renal capsule of adult mice as previously described (Ferguson and Parrott, 1972). Isografts were prepared between CBA mice; allografts by implanting foetal colon 
from Balb/c mice into the CBA hosts. Pregnant mice were killed on the 19th day of gestation and graft age was calculated from the day of transplantation (day 0). The age of normal mice was calculated from the day of birth (day 1). Three groups of tissue were studied-normal colon taken from groups of mice aged 5, 10, 14, 18, 21, 24, 42, and 120 days; colonic isografts aged $3,7,14,21,28$, and 120 days; colonic allografts aged 3,5 , and 7 days after transplantation. The numbers of colons and grafts examined and the number of satisfactory crypt preparations obtained for these are shown in the Table.In each experimental group, measurements were taken from five or more specimens.

Table Number of normal colon isografts and allografts studied and number and percentage of these giving satisfactory crypt squash preparations

\begin{tabular}{llcl}
\hline & $\begin{array}{l}\text { Grafts/colon } \\
\text { examined }\end{array}$ & $\begin{array}{l}\text { Satisfactory } \\
\text { crypt squash }\end{array}$ & $(\%)$ \\
\hline Normal colon & 56 & 48 & 86 \\
Isografts & 48 & 39 & 81 \\
Allografts & 24 & 18 & 75 \\
Total & 128 & 105 & 82 \\
\hline
\end{tabular}

CRYPT METAPHASE ACCUMULATION

The animal was given colchicine, $5 \mathrm{mg} / \mathrm{kg}$ body weight, intraperitoneally at an accurately timed interval (one to two hours) before it was killed by an overdose of ether. The colon, or part of the kidney with the attached graft, was dissected out and metaphase accumulation measured as follows.

The tissues were fixed in Clark's fixative (three parts absolute ethanol and one part glacial acetic acid) for 24 hours. Specimens were then transferred from the fixative into $70 \%$ alcohol for storage until the time of examination. Square pieces $0.5 \times 0.5 \mathrm{~cm}$ were cut from normal colon, and isografts and allografts were dissected free of adjacent kidney, trimmed, and processed whole. The fragments were stained in bulk by a modification of the Feulgen reaction-they were transferred to $50 \%$ ethanol for 10 minutes, then to water for 10 minutes, to normal $\mathrm{HCl}$ for six minutes at $60^{\circ} \mathrm{C}$, washed in tap water, stained with Schiff's reagent for 20 minutes, and finally washed again in tap water. Preparations of individual crypts were made by cutting thin slivers of colon $0.5 \mathrm{~mm} \times 1.0 \mathrm{~mm}$ using a dissecting microscope. In some isografts adherent intraluminal mucus had to be removed gently before this could be done. These strips were then placed edge-on onto a microscope slide, covered with a drop of $45 \%$ acetic acid, and gently squashed beneath a coverslip. The blocked metaphases in each crypt were then counted using an eyepiece grid at a magnification of $\times 400$. A minimum of 10 crypts was counted for each preparation, two preparations were made from each specimen of colon, and the 20 values were meaned. Metaphase accumulation per hour was calculated from the formula $\frac{m}{t} \times 60$, where $m$ is the number of metaphases per crypt, and $t$ is the time in minutes between colchicine injection and death. To negate any effects of possible diurnal variation in the turnover of surface cells in mouse colon all mice were killed between $11 \mathrm{am}$ and $1 \mathrm{pm}$.

\section{CRYPT LENGTH}

Grafts of foetal colon and normal mouse colon, in similar categories to those described above were fixed in neutral formal saline, embedded in paraffin wax, and $4 \mu$ histological sections cut perpendicular to the mucosal surface. Sections were stained with haematoxylin and eosin and examined on a magnification of $\times 400$. The lengths of 20 crypts in each section were measured with a micrometer eyepiece, and six colons or grafts were used for each experimental group.

STATISTICAL ANALYSIS

Comparison between groups was carried out by using analysis of variance and Student's $t$ test.

\section{Results}

Allografts and isografts grew satisfactorily, as described in previous reports (Ferguson and Parrott, 1972; Holden and Ferguson 1976; MacDonald and Ferguson, 1977). Isografts grew progressively larger after implantation; allografts grew for seven to 10 days before rejection. The technique of crypt dissection was easily applied to normal colon, and grafts, though more difficult to examine, could, with care, be satisfactorily processed. As can be seen from the Table, it was necessary to discard some $25 \%$ of the grafts, almost exclusively those less than 7 days old, because crypts were destroyed rather than simply separated during squashing. Similar losses of tissue occurred in all three experimental groups.

\section{METAPHASE ACCUMULATION IN NORMAL COLON}

At 5 days after birth, metaphase accumulation rate was $2 \cdot 25$ per crypt per hour and there was no significant change in this rate up to the age of 18 days, although crypts became progressively longer during this time $(P<0.01)$ (Fig. 1). Between the 18th and 21 st days (shortly after the time of weaning in the mouse) metaphase accumulation rose sharply, there being significant differences between the values for 
18 and 21 , and 21 and 24 days $(P<0.01)$. After 24 days, the rate of metaphase accumulation reached a plateau.

METAPHASE ACCUMULATION IN ISOGRAFTS OF MOUSE COLON

A different pattern of metaphase accumulation rates was found in isografts (Fig. 1). Metaphase accumulation rate was low, 1.9 per crypt per hour on day 3 and similar values were found throughout the first two weeks after transplantation. Just as is the case in normal colon, there was significant lengthening of graft colonic crypts during this time $(P<0.01)$. Metaphase accumulation rates were slightly higher in the specimens taken at day 21 (3.5 per crypt per hour) and day 28 (4.3 per crypt per hour) but differences from the earlier groups were not statistically significant. When metaphase accumulation rates in normal colonic crypt and in isografts were compared, significant differences were found at 21 days, $24 \mathrm{v} 28$ days, and at 120 days $(\mathrm{P}<0.01)$.

METAPHASE ACCUMULATION IN

ALLOGRAFTS OF MOUSE COLON

Colonic allografts damaged by the cell-mediated immune reaction of rejection yielded satisfactory crypt preparations only up to 7 days after transplantation. Rates of metaphase accumulation at 3,5 , and 7 days were all significantly greater than in isografts of similar age $(P<0.01)$. Despite the higher cell production, the crypt length did not rise between 3 and 7 days (Fig. 2).

\section{Discussion}

The technique of metaphase arrest with microdissection of the mucosa allows examination of the kinetic properties of single intestinal crypts and we have found that the technique which was described for use in the small intestine can be readily adapted to examine cell kinetics in colonic epithelium. The method is applicable to normal colon of animals at any age and its application to grafts of foetal mouse colon has allowed definition of the effects of several factors on cell kinetics. The experimental situations examined here-of normal post-natal growth, of antigen-free growth in isografts, and the effects of cell-mediated immunity-parallel those examined in a previous morphological study (Holden and Ferguson 1976). The results highlight the fact that there is no invariable or direct relationship between crypt cell production and crypt length.

Experiments described in this paper have shown that normal baby mouse colon produces two to three cells per crypt per hour, a similar rate of cell production to that seen in small intestine (MacDonald, 1974). In the first two weeks of life crypts
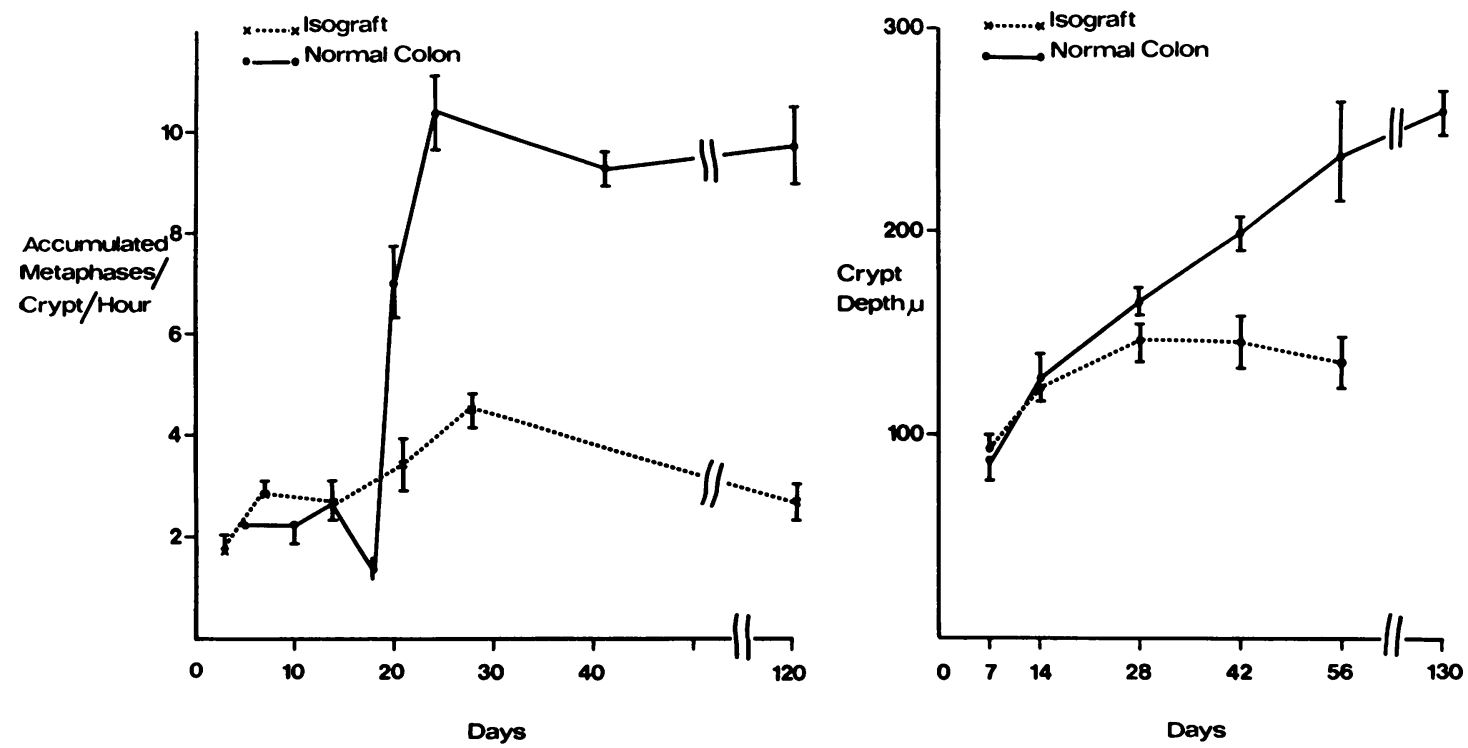

Fig. 1 Metaphase accumulation per crypt per hour and crypt length in normal colon. Left: metaphase accumulation per hour (ordinate) at different times in days (abscissa), after birth (normal colon) and transplantation (isografts). Each point is mean of a group $\pm S E M$. Right: crypt depth ( $\mu$, ordinate) at various times (days, abscissa); mean \pm 1 SEM. 

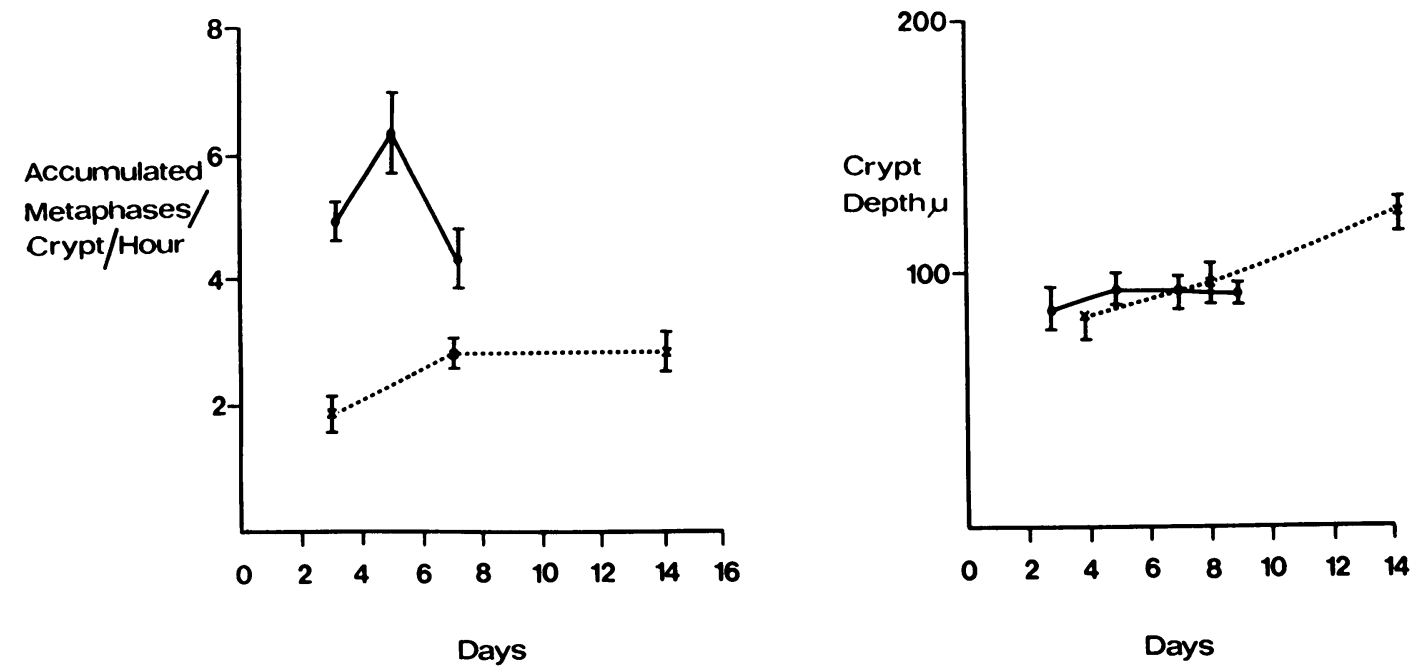

Fig. 2 Accumulated metaphases per crypt per hour and crypt depth for colonic isografts $(\times \ldots \ldots \times)$ and allografts (-—). Left: metaphase accumulation per crypt per hour (ordinate) at different times in days (abscissa), after transplantation; mean \pm SEM. Right: crypt depth ( $\mu$, ordinate) at various times (days, abscissa); mean $\pm S E M$.

steadily increased in length, and it is likely that many of the newly formed cells remain in the crypt to expand its size. After weaning, between the 18th and 21 st days after birth, there was a sharp increase in colonic mitotic activity but during this time colonic crypts continued to lengthen steadily at the same rate as previously. Similar changes have been noted to occur in crypt mitotic activity in the small bowel (MacDonald, 1974). Changes occurring in the intestinal mucosa about the time of weaning may reflect a phase of the intrinsic development of the tissue, perhaps mediated by hormonal changes, or may result from changes in the external environment with the introduction of bacterial and food antigen into the gut lumen. Antigen-free grafts of intestine provide a suitable model for differentiating between intrinsic and extrinsic stimuli and, as the sharp increase in crypt cell production does not occur in such grafts of colon, as here, or of small bowel (MacDonald, 1974), it is likely that these kinetic changes reflect changes in the external environment occurring at the time of weaning.

Several mechanisms could alter epithelial cell kinetics. There is an increase in the bulk of the colonic contents which occurs together with changes in microbial flora, changes which may damage the surface mucosa and so induce increased cell production. In adult rats, dietary bulk has been reported to initiate colonic mucosal hyperplasia (Dowling et al., 1967; Stragand and Hagemann, 1977). Alternatively, immune reactions between mucosal lymphoid tissues and antigens present in food or bacteria in the bowel lumen may be the initiating factor causing the rise in cell turnover. The colonic isografts, which do not show the kinetic changes seen in normal bowel, distend progressively with mucus. This might suggest that distension is not the major mechanism responsible for the burst of activity at weaning; however, clearly there are important differences between the distension of normal rat colon by a bulk diet and the distension which occurs because of the continuous accumulation of mucus in the closed system of an isograft. A number of factors, both extrinsic and intrinsic, may be involved in the production of the kinetic changes seen in developing colon and our findings highlight the need for further research in this area of gut physiology.

The effects of local cell-mediated immune reactions on epithelial cell kinetics have been studied in the small intestine of mice using the models of allograft rejection (Ferguson and Parrot, 1973; MacDonald and Ferguson 1976), and graft v. host disease (MacDonald and Ferguson, 1977; Ferguson and MacDonald, 1977). MacDonald and Ferguson (1977) have suggested that the cytokinetic features which accompany the morphological changes of lymphocyte infiltration, crypt hyperplasia, and villus atrophy in the small bowel occur in two phases. In the first phase, increased crypt mitotic activity occurs with accelerated cell transit along the sides of the villi and in this first phase the villi remained normal in length. This is followed by phase two in which high cell production continues in the crypt 
but is accompanied by greatly accelerated cell loss with destruction of the villi. They suggested that the mechanism of villus destruction is not by damage to the enterocytes themselves but is a result of damage to and collapse of the underlying stroma of the villi.

In the colon, examination by routine histopathological techniques suggested that the features of colonic rejection were rather different from those of the small bowel. There was lymphocyte infiltration but with a reduction in the number of mucous cells and with shortening of the crypts as compared with crypts of isografts of similar age (Holden and Ferguson, 1976). However, the cell kinetic studies have now shown that the short allograft crypts have a two- to three-fold greater rate of cell production than occurs in isografts or normal intestine of the same age. These changes may be interpreted by using the same conceptual model as proposed for the small intestine.

The arrangement of epithelial cell compartments within the colonic crypt is basically similar to that seen in the villus and crypt of small bowel. In the base of the crypt is the progenitor cell region where mitotic activity continues. Above this there is a compartment where cells undergo functional maturation and then enter the mature cell compartment. In the small bowel the mature cell compartment covers the villi. In the colon it is represented by the cells at the very mouth of the crypt and in the short length of surface mucosa which abuts against the surface mucosa rising from the adjacent crypts. From this inter-crypt junction cell loss into the colonic lumen occurs in the same way as from the villus tip in the small bowel. In the normal small bowel, mucosal thickness is made up by the length of villus plus crypt. In normal large bowel, mucosal thickness is the same as the length of the crypt. Increased cell production with expansion of the progenitor cell region and loss of the entire mature cell compartment may occur in the small bowel to give the appearance of crypt hyperplasia and villus atrophy yet total mucosal thickness which is similar to or less than that in normal small bowel. Similar expansion of the progenitor cell region in the colonic crypts, together with loss of the mature cell compartment, results in a shorter crypt than normal and so a thinner mucosa than normal, as illustrated in Fig. 3. The phase 1, shown in Fig. 3, and corresponding to the phase 1 described in the small bowel by MacDonald (1974), is hypothetical and has not been observed experimentally.

Conventional examination of rectal and colonic mucosa in diseased states has rarely demonstrated striking changes in crypt morphology. However, after severe and recurrent attacks of ulcerative colitis, there may be atrophy of the mucosa with reduced numbers of short and branching crypts. Autoradiographic studies of cultured rectal mucosa in ulcerative colitis have suggested an increase in crypt cell production in this disease (Eastwood and Trier, 1973; Bleiberg and Galand, 1974). Although, as we have previously pointed out (Holden and Ferguson, 1976), the morphological changes of the colonic rejection model did not closely parallel those of ulcerative colitis, the changes in the model

\section{LOCAL C.M.I. REACTION}

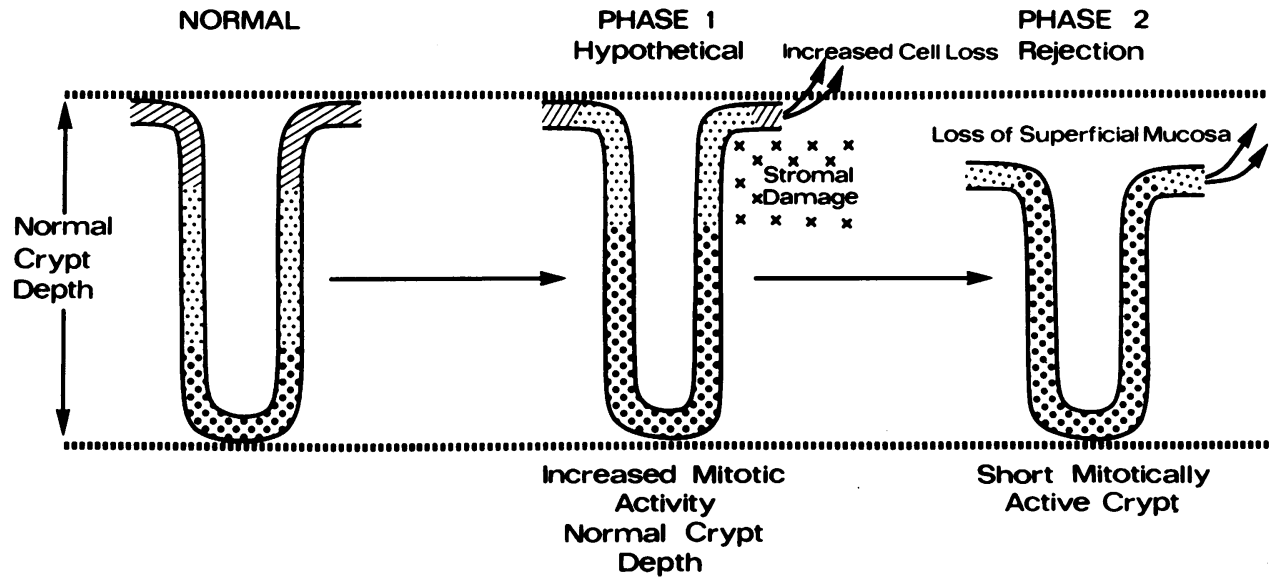

Fig. 3 Effects of a local cell-mediated immune reaction on colonic architecture. Diagram shows the temporal progression of morphological changes during a cell-mediated immune reaction from normal on the left to the mucosa with short crypts on the right. Large dots: progenitor cell compartment. Small dots: maturation compartment. Hatched lines: mature cell compartment. 
suggest possibly that a state of accelerated cell production with rapid transit of cells from crypt to surface mucosa can result in immaturity of the surface epithelium. A functionally diseased intestinal mucosa may be present, although, by light microscopy, mucosal architecture is normal or close to normal. The microdissection method described in this paper could be modified for the study of colonic and rectal biopsies and allow formal examination of this hypothesis; a modification of the technique for use with small intestinal biopsies has already been reported (Ferguson et al., 1977).

\section{References}

Bleiberg, H., and Galand, P. (1974). Mesures autoradiographiques de la proliferation cellulaire dans le tube digestif. Acta Gastroenterologica Belgica, 37, 475-487.

Clarke, R. M. (1971). A comparison of metaphase arresting agents and tritiated thymidine autoradiography in measurement of the rate of entry of cells into mitosis in the crypts of Lieberkühn of the rat. Cell and Tissue Kinetics, 4, 263-272.

Dowling, R. H., Riecken, E. O., Laws, J. W., and Booth, C. C. (1967). The intestinal response to high bulk feeding in the rat. Clinical Science, 32, 1-9.

Eastwood, G. L., and Trier, J. S. (1973). Epithelial cell renewal in cultured rectal biopsies in ulcerative colitis. Gastroenterology, 64, 383-390.

Ferguson, A., and MacDonald, T. T. (1977). Effects of local delayed hypersensitivity on the small intestine. In Immunology of the Gut, pp. 305-327 (Ciba Foundation Symposium, New Series, 46). Elsevier North-Holland.

Ferguson, A., and Parrott, D. M. V. (1972). Growth and development of 'Antigen-Free' grafts of foetal mouse intestine. Journal of Pathology, 106, 95-101.

Ferguson, A., and Parrott, D. M. V. (1973). Histopathology and time course of rejection of allografts of mouse small intestine. Transplantation, 15, 546-554.

Ferguson, A., Sutherland, A., MacDonald, T. T., and Allan, F. (1977). Technique for micro-dissection and measurement in biopsies of human small intestine. Journal of Clinical Pathology, 30, 1068-1073.

Holden, R. J., and Ferguson, A. (1976). Histopathology of cell mediated immune reaction in mouse colon-allograft rejection. Gut, 17, 661-670, 1976.

Lipkin, M. (1973). Proliferation and differentiation of gastro-intestinal cells. Physiological Reviews, 53, 891-915.

MacDonald, T. T. (1974). Delayed Hypersensitivity Reactions in the Small Intestine. Ph.D. Thesis, University of Glasgow.

MacDonald, T. T., and Ferguson, A. (1976). Hypersensitivity reactions in the small intestine. Two effects of allograft rejection on mucosal architecture and lymphoid cell infiltrate. Gut, 17, 81-91.

MacDonald, T. T., and Ferguson, A. (1977). Hypersensitivity reactions in the small intestine. The effects of allograft rejection and of graft-versus-host disease on epithelial cell kinetics. Cell and Tissue Kinetics, 10, 301-312.

Stragand, J. J., and Hagemann, R. F. (1977). Effects of lumenal contents on colonic cell replacement. American Journal of Physiology, 233, E208-E211. 